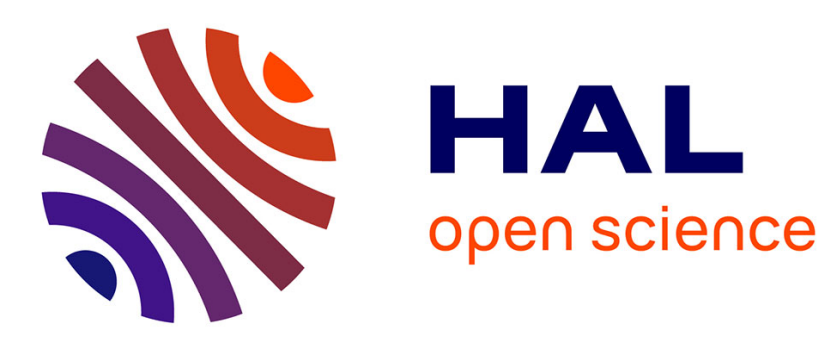

\title{
Embedding finite and infinite words into overlapping tiles
}

\author{
Anne Dicky, David Janin
}

\section{To cite this version:}

Anne Dicky, David Janin. Embedding finite and infinite words into overlapping tiles. Developments in Language Theory (DLT), Aug 2014, Ekaterinburg, Russia. pp.339-347, 10.1007/978-3-319-096988_30. hal-00910002v2

\section{HAL Id: hal-00910002 \\ https://hal.science/hal-00910002v2}

Submitted on 15 May 2014

HAL is a multi-disciplinary open access archive for the deposit and dissemination of scientific research documents, whether they are published or not. The documents may come from teaching and research institutions in France or abroad, or from public or private research centers.
L'archive ouverte pluridisciplinaire HAL, est destinée au dépôt et à la diffusion de documents scientifiques de niveau recherche, publiés ou non, émanant des établissements d'enseignement et de recherche français ou étrangers, des laboratoires publics ou privés. 
Embedding finite and infinite words into overlapping tiles

May 15, 2014

Anne Dicky, David Janin

LaBRI, Université de Bordeaux, 351, cours de la Libération, F-33405 Talence, FRANCE \{dickyljanin\}@labri.fr 



\title{
Embedding finite and infinite words into overlapping tiles
}

\author{
Anne Dicky, David Janin \\ LaBRI, IPB, Université de Bordeaux, \\ 351, cours de la Libération, \\ F-33405 Talence, FRANCE \\ \{dickyljanin\}@labri.fr
}

\begin{abstract}
In this paper, we study languages of finite and infinite birooted words. We show how the embedding of free $\omega$-semigroups of finite and infinite words into the monoid of birooted words can be generalized to the embedding of two-sorted $\omega$-semigroups into (some notion of) onesorted ordered $\omega$-monoids. This leads to an algebraic characterization of regular languages of finite and infinite birooted words that generalizes and unifies the known algebraic characterizations of regular languages of finite and infinite words ${ }^{1}$.
\end{abstract}

\section{Introduction}

Infinite strings naturally arise in Software Engineering as models of (potentially) non-terminating system behaviors. From an abstract point of view, infinite strings are defined as infinite concatenations of non-empty finite strings, with an infinite associativity law ensuring that this infinite product is compatible with the standard concatenation of finite strings. Such an infinite string is depicted Figure 1. The resulting algebraic structures are the (free) $\omega$-semigroups [30],

\begin{tabular}{|l|l|l|l|l|l|l}
\hline$a b c$ & $d a$ & $b a b a$ & $b a b$ & $a b$ & $a b a b$ & \\
\hline
\end{tabular}

Fig. 1. An infinite product for $a b c d(a b)^{\omega}$.

that is, the free semigroups of finite strings with string concatenation as product extended with the infinite product to produce infinite strings and a related mixed product to concatenate finite strings in front of infinite strings. This leads to the notions of $\omega$-semigroups and morphisms that provide an algebraic characterization of regular languages of infinite words (see [30]). These results also offer quite a deep insight of the mathematical properties of Büchi's automata on infinite strings [3] and the associated algorithmic tools that have been developed for model-checking and program verification (see e.g. [9] for an overview).

\footnotetext{
1 This report is the long version of [5]
} 
Programming languages such as Haskell [12] allow for effectively defining infinite streams of values by means of lazy evaluation mechanisms. In view of application to temporal media programming, that is, finite and infinite sequences of media type values such as images, sounds or control events, the abstract data type implicitly induced by $\omega$-semigroups is enriched with the parallel product of finite or infinite strings. This leads to effective tools for handling temporal media types [10]. The notion of parallel product is depicted Figure 2.

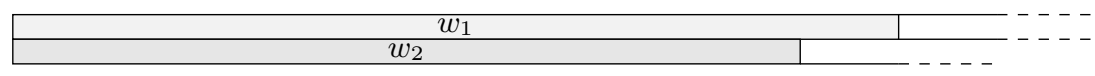

Fig. 2. A parallel product of two words.

It has recently been advocated that there are benefits in embedding finite strings as well as infinite streams into (some notion of) tiled temporal media [13]. Typical temporal media synchronization constructs such as musical pickups (anacrusis) lead to distinguishing the effective starts of temporal media - the first note of a melody, from their logical starts - the first strong beat of that melody [1]. Then, the notion of tiled temporal media allows for a fairly simple modeling of these multi-level synchronization constructs. This comes from the fact that they are equipped with a tiled product that is neither a sequential nor a parallel product but both $[21,13]$.

More precisely, distinguishing string values from strings synchronization usage can be modeled by enriching the string data type by means of two synchronization marks: the pre synchronization mark or input root, and, respectively, the post synchronization mark or or output root (see Figure 3). These synchro-

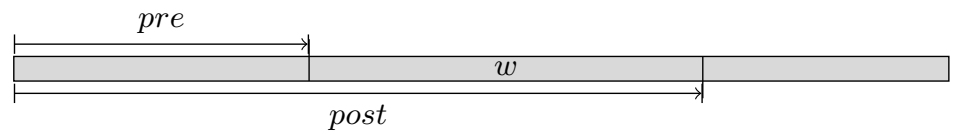

Fig. 3. A tiled temporal media (or birooted word).

nization marks tell how such enriched words are to be positioned with respect to the temporal media that comes before, or, respectively, after, the current temporal media. The resulting objects, here called birooted words, are then equipped with a single product, the tiled product, that is neither a sequential product as string concatenation, not a parallel product as string "zip", but both (see Figure 4 and [13] for a fairly simple implementation of these ideas on top of the musical library Euterpea [11] in Haskell).

In the general setting of higher-dimensional strings and tiling semigroups [22, $23,20]$, the tiled temporal media and the related tiled product lay in the mathematical framework of inverse semigroup theory [25] and semigroups with local 


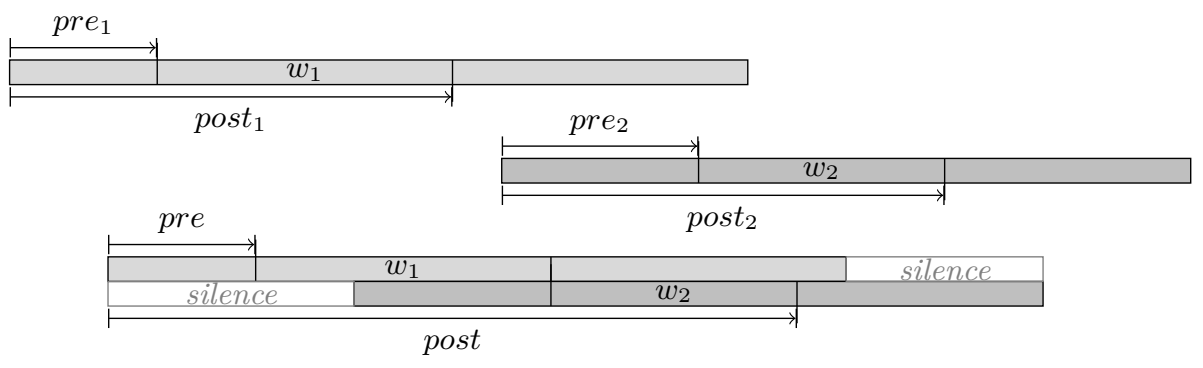

Fig. 4. A tiled product instance

units $[24,7,8]$. Projecting tiled temporal media onto semantical tags, we obtain sorts of tiled words. In the finite case, the induced algebra is the inverse semigroup of McAlister whose elements are birooted words [27, 26].

Aiming at providing a robust mathematical framework for handling languages of tagged tiled temporal media, our purpose here is to extend the languagetheoretical tools available for languages of finite birooted words (see $[17,15,4$, 18]) to the case of infinite birooted words.

For such a purpose, we show that the obvious embedding of the free $\omega$ semigroups of finite and infinite words into the monoid of birooted words can be generalized to the embedding of two-sorted $\omega$-semigroups into one-sorted ordered $\omega$-monoids.

This leads to an algebraic characterization of regular languages of finite and infinite birooted words that generalizes and unifies the algebraic characterizations of regular languages of finite and infinite words.

For the sake of simplicity, we restrict our presentation to one-sided birooted words. The resulting monoid turned out to be the filter completion (with respect to the natural order [25]) of the polycyclic monoid of Perrot and Nivat [29]. However, all proposed concepts and constructions can clearly be extended to two-sided birooted words, that is, within the filter completion of the submonoid of McAlister [25] of positive tiles. It follows that our results also apply to biinfinite words with the resulting ordered $\omega$-monoids equipped with both a left and a right infinite product.

Technically, our results capitalize on the quasi-inverse expansion of semigroups presented in $[15,16]$. However, in the settings of infinite bi-rooted words, a congruence property, that appears in the notion of Ehresmann semigroups [24] is essential and was totally left unnoticed (and unused) in the finite case. The resulting algebraic framework is thus a refinement of the one proposed in [15, $14]$.

\section{From finite or infinite words to birooted words}

In this section we define the monoid of positive finite or infinite birooted words. From a mathematical point of view, that monoid is a submonoid of the filter 
completion of the monoid of McAlister [26]. The language theory of the McAlister monoid has been developed in $[17,15,18,4]$ for finite tiles.

Let $A$ be a finite alphabet. Let $\left(A^{*}, \cdot\right)$ be the free monoid of finite strings on the alphabet $A$, and let $\left(A^{+}, A^{\omega}, \cdot, *, \pi\right)$ be the associated free $\omega$-semigroup (see [30]) with finite product $\cdot$, mixed product $*$ and infinite product $\pi$.

In the sequel, both the finite product $u_{1} \cdot u_{2}$ when $u_{1}, u_{2} \in A^{*}$ or the mixed product $u_{1} * u_{2}$ when $u_{1} \in A^{*}$ and $u_{2} \in A^{\omega}$ may simply be denoted by $u_{1} u_{2}$.

The set $A^{\infty}=A^{*} \cup A^{\omega}$ of finite and infinite strings is ordered by the prefix order $\leq_{p}$, defined, for every $u$ and $v \in A^{\infty}$, by $u \leq_{p} v$ when either $u=v$, or $u$ is finite and there is $w \in A^{\infty}$ such that $v=u w$. Extended with a maximum element denoted by 0 , the set $A^{\infty}+0$ ordered by the prefix order $\leq_{p}$ is a complete lattice. The prefix join $u \vee_{p} v$ of two words $u$ and $v \in A^{\infty}$ is then the least word $w \in A^{\infty}$, if it exists, such that we have both $u \leq_{p} w$ and $v \leq_{p} w$, or 0 otherwise. Then, for every $u \in A^{*}$ and $v \in A^{\infty}$, the right residual $u^{-1}(v)$ of $v$ by $u$, is defined as the word $w \in A^{\infty}$, unique if it exists, such that $v=u w$. We take $u^{-1}(v)=0$ otherwise. By definition, $u^{-1}(v) \neq 0$ if and only if $u \leq_{p} v$.

Definition 1. A positive (right) birooted word $u$ is a pair $u=\left(u_{1}, u_{2}\right)$ where $u_{1} \in A^{*}$ and $u_{2} \in A^{\infty}$. The word $u_{1} u_{2} \in A^{\infty}$ is called the domain of the birooted word $u$, and the word $u_{1}$, its root path. The birooted word $\left(u_{1}, u_{2}\right)$ is finite when $u_{2}$ is finite. The set of positive birooted words on the alphabet $A$ is denoted by $T^{\infty}(A)$. The set of finite positive birooted words on $A$ is denoted by $T^{+}(A)$.

The product $u \cdot v$ of two birooted words $u=\left(u_{1}, u_{2}\right)$ and $v=\left(v_{1}, v_{2}\right)$ is defined as the birooted word $w=\left(w_{1}, w_{2}\right)$ with $w_{1}=u_{1} v_{1}$ and $w_{2}=v_{1}^{-1}\left(u_{2}\right) \vee_{p} v_{2}$ when $v_{1}^{-1}\left(u_{2}\right) \vee_{p} v_{2} \neq 0$. Otherwise, we take $u \cdot v=0$ for some new birooted word 0 , with $0 \cdot u=u \cdot 0=0$ for every $u \in T^{\infty}(A)$, and $0 \cdot 0=0$.

Examples. The following examples illustrate the definition of the product:

$$
\begin{array}{ll}
(a b, a b) \cdot(a, b c)=(a b a, b c) & \left(a b,(a b)^{\omega}\right) \cdot(a, b c)=0 \\
\left(a b,(a b)^{\omega}\right) \cdot(a, b a)=\left(a b a,(b a)^{\omega}\right) & (a b, a b) \cdot\left(a,(b a)^{\omega}\right)=\left(a b a,(b a)^{\omega}\right) \\
(a b, a b) \cdot\left(a,(b c)^{\omega}\right)=\left(a b a,(b c)^{\omega}\right) & (a b, a c) \cdot\left(a,(b a)^{\omega}\right)=0 \\
(1, a b) \cdot(1, a b c)=(1, a b c) & (1, a b) \cdot(1, a c)=0
\end{array}
$$

Let $T_{0}^{\infty}(A)$ (resp. $T_{0}^{+}(A)$ ) be the set of positive birooted words (resp. finite positive birooted words) extended with 0 .

Theorem 2. The set $T_{0}^{\infty}(A)$ equipped with the above product is a partially ordered monoid with unit $1=(1,1)$.

Proof. Easily proved by routine arguments.

Let $u \mapsto u^{R}$ and, resp., $u \mapsto u^{L}$ be the right projection (or reset) and, resp., the left projection (or co-reset) defined on the monoid $T_{0}^{\infty}(A)$ by

$$
0^{R}=0^{L}=0, \quad u^{R}=\left(1, u_{1} u_{2}\right) \quad \text { and } \quad u^{L}=\left(1, u_{2}\right)
$$


for every $u=\left(u_{1}, u_{2}\right) \in T_{0}^{\infty}(0)$. Then the natural order relation (see [24,14]) is defined by

$$
u \leq v \quad \text { when } \quad u=u^{R} \cdot v \cdot u^{L}, \quad \text { or, equivalently, } \quad u=u^{R} \cdot v
$$

for every $u$ and $v \in T_{0}^{\infty}(A)$. Elements $u$ such that $u \leq 1$ are called subunits and the set of subunits is denoted by $U\left(T_{0}^{\infty}(A)\right)$.

Theorem 3. The natural order relation on $T_{0}^{\infty}(A)$ is a partial order relation stable under product, that is if $u \leq v$ then $u w \leq v w$ and $w u \leq w v$ for all $u, v, w \in T_{0}^{\infty}(A)$. Subunits and idempotents coincide, that is, we have $u \leq 1$ if and only if $u=u u$ for all $u \in T_{0}^{\infty}(A)$. Moreover, the set $U\left(T_{0}^{\infty}(A)\right)$ ordered by the natural order is a complete lattice with product as meet.

Proof. All statements can directly be proved by routine arguments. More interestingly, on can check that the set $T_{0}^{\infty}(A)$ of (right) birooted words is indeed isomorphic to the filter completion of the set $T_{0}^{+}(A)$ of one-sided finite birooted words ordered by the natural order, or, equivalently, the polycyclic monoid of Nivat and Perrot[29].

More precisely, an (order) filter is a non empty subset $X$ of $T_{0}^{+}(A)$ that is upward closed, i.e. for every $u$ and $v \in T_{0}^{+}(A)$, if $u \leq v$ and $u \in X$ then $v \in X$, and downward directed, i.e. for every $u$ and $v \in X$ there exists $w \in X$ such that $w \leq u$ and $w \leq v$. The set $\mathcal{F}\left(T^{+}(A)\right)$ of filters is equipped with the product defined, for every filters $X$ and $Y$, by $X \cdot Y=\left\{w \in T_{0}^{+}(A): \exists u \in X, v \in Y, u \cdot v \leq\right.$ $w\}$ that is well defined since the natural order is stable under product.

Then, one can show that the mapping $\varphi: T^{\infty}(A) \rightarrow \mathcal{F}\left(T^{+}(A)\right)$ that maps every bi-rooted word $u \in T^{\infty}(A)$ to the set of finite birooted words $\varphi(u)=$ $\left\{v \in T^{+}(A): u \leq v\right\}$ is an isomorphism from $T^{\infty}(A)$ to the monoid of filters of $T^{+}(A)$.

Remark. In the finite case $[17,15]$, we consider positive two-sided birooted words. Here, in a tile $\left(u_{1}, u_{2}\right) \in T_{0}^{\infty}(A)$, we always have empty domain on the left of the root path: the birooted words defined here are one-sided. A two-sided positive birooted word would be a triple $\left(u_{1}, u_{2}, u_{3}\right)$ with a finite or infinite left word $u_{1} \in{ }^{\infty} A$, a finite root path $u_{2} \in A^{*}$, and a finite or infinite right word $u_{3} \in A^{\infty}$. All definitions and results presented here are easily extended to the two-sided case.

An immediate interest of the notion of finite and infinite birooted words is that the (two sorted) free omega monoid $\left(A^{+}, A^{\omega}\right)$ can be embedded into the (one sorted) monoid $T_{0}^{\infty}(A)$, that is, the pair $\left(T_{0}^{\infty}(A), U\left(T_{0}^{\infty}(A)\right)\right)$ can be equipped with a mixed and an infinite product in such a way that it is an $\omega$ semigroup into which the free $\omega$-semigroup $\left(A^{+}, A^{\omega}\right)$ can be embedded. More precisely, let $u * v$ be the mixed product defined by $u * v=(u \cdot v)^{R}$ for all $u \in T_{0}^{\infty}(A)$ and $v \in U\left(T_{0}^{\infty}(A)\right)$, and let $\pi\left(u_{i}\right)_{i \in \omega}$ be the infinite product defined by $\pi\left(u_{i}\right)_{i \in \omega}=\bigwedge_{n \in \omega}\left(u_{0} \cdot u_{1} \cdots \cdot u_{n-1}\right)^{R}$ for all infinite sequences of birooted words $\left(u_{i}\right)_{i \in \omega}$. Then we have: 
Theorem 4. The pair $\left(T_{0}^{+}(A), U\left(T_{0}^{\infty}(A)\right)\right)$ equipped with the finite product, the mixed product and the infinite product is a well-defined $\omega$-semigroup.

Moreover, let $\theta_{f}: A^{*} \rightarrow T_{0}^{+}(A)$ be the mapping defined by $\theta_{f}(v)=(v, 1)$ for every finite word $v \in A^{*}$ and let $\theta_{\omega}: A^{\omega} \rightarrow U\left(T_{0}^{\infty}(A)\right)$ be the mapping defined by $\theta_{\omega}(w)=(1, w)$ for every infinite word $w \in A^{\omega}$.

Then, the pair of mappings $\left(\theta_{f}, \theta_{\omega}\right):\left(A^{+}, A^{\omega}\right) \rightarrow\left(T_{0}^{+}(A), U\left(T_{0}^{\infty}(A)\right)\right)$ is an $\omega$-semigroup embedding, that is, a one-to-one mapping that preserves the finite, the mixed and the infinite products.

Proof. Clearly, the infinite product is well-defined since $U\left(T_{0} \infty\right)$ ordered by the natural order is a complete lattice with product as meet. Then, it is routine to check that the pair $\left(T_{0}^{+}(A), U\left(T_{0}^{\infty}(A)\right)\right.$ with product $\cdot$, mixed product $*$ and infinite product $\pi$ is an $\omega$-semigroup. Moreover, $\theta_{f}$ is clearly a one-to-one monoid morphism and $\theta_{\omega}$ is one-to-one and, moreover, for every $v \in A^{*}$ and $w \in A^{\omega}$, we easily check that we have $\theta_{\omega}(v * w)=\left(\theta_{f}(v) \cdot \theta_{\omega}(w)\right)^{R}$. Then it is routine to check that $\theta_{\omega}(v * w)=\theta_{f}(v) * \theta_{\omega}(w)$ for all $v \in A^{*}$ and $w \in A^{\omega}$ and that

$$
\theta_{\omega}\left(\pi\left(u_{i}\right)_{i \in \omega}\right)=\pi_{i \in \omega} \theta_{f}\left(u_{i}\right)
$$

for every $\left(u_{i}\right)_{i \in \omega} \in\left(A^{+}\right)^{\omega}$. In other words, the pair of mappings $\left(\theta_{f}, \theta_{\omega}\right)$ is an $\omega$-semigroup morphism.

In the particular case where the infinite sequence $\left(u_{i}\right)_{i \in \omega}$ is constant, that is, when there is some $v \in T_{0}^{\infty}(A)$ such that $u_{i}=v$ for every $i \in \omega$, then we write $v^{\omega}$ for $\pi\left(u_{i}\right)_{i \in \omega}$. One can easily check that for every idempotent (or subunit) $u \leq 1$ we have $u^{\omega}=u$.

\section{$3 \quad$ Embedding $\omega$-semigroups into ordered $\omega$-monoids}

We show here that the above embedding of the free $\omega$-semigroup $\left(A^{+}, A^{\omega}\right)$ into the monoid of birooted words $T^{\infty}(A)$ can be generalized to an embedding of any $\omega$-semigroup $S$ into (some notion of) ordered $\omega$-monoid $M(S)$.

Let $M$ be a monoid partially ordered by a relation $\leq$. We assume that the order relation $\leq$ is stable under product, i.e. if $x \leq y$ then $x z \leq y z$ and $z x \leq z y$ for every $x, y$ and $z \in M$. The set $U(M)$ of subunits of the partially ordered monoid $M$ is defined by $U(M)=\{y \in M: y \leq 1\}$.

The following definition is adapted from [18] and then, following [24], refined with the congruence property $[14,6]$.

Definition 5 (Adequately ordered and E-ordered monoids). A (stable) partially ordered monoid $M$ is an adequately ordered monoid when:

(A1) idempotent subunits: for every $x \in M$, if $x \leq 1$ then $x x=x$,

(A2) left and right projection: for every $x \in M$, both the left projection defined by $x^{L}=\min \{y \in U(M): x y=x\}$ and the right projection defined by $x^{R}=\min \{y \in U(M): y x=x\}$ exist in $U(M)$,

It is an Ehresmann-ordered monoid (or E-ordered monoid) when, moreover: 
(A3) congruence property: for every $x, y, z \in M$, if $x^{L}=y^{L}$ then $(x z)^{L}=(y z)^{L}$ and if $x^{R}=y^{R}$ then $(z x)^{R}=(z y)^{R}$,

Examples. Every monoid trivially ordered is an adequately ordered monoid. Every inverse monoid ordered by the natural order [25] is also an adequately ordered monoid with left and right projections defined by $x^{R}=x \cdot x^{-1}$ and $x^{L}=x^{-1} \cdot x$ for every element $x$.

The notion of adequately ordered monoid is extended here with an infinite (right) product as follows.

Definition 6 (E-ordered $\omega$-monoid). An E-ordered $\omega$-monoid if an E-ordered monoid $M$ equipped with an infinite product operator $\pi: M^{\omega} \rightarrow U(M)$ satisfying the following properties:

(I1) subunit preservation: for every $\left(x_{i}\right)_{i \in \omega}$ such that for every $i \in \omega$ we have $x_{i}=x$ for some $x \in U(M)$, then $\pi\left(x_{i}\right)_{i \in \omega}=x$,

(I2) monotonicity: for every infinite sequences $\left(x_{i}\right)_{i \in \omega} \in M^{\omega}$ and $\left(y_{i}\right)_{i \omega}$, if $x_{i} \leq y_{i}$ for every $i \in \omega$ then $\pi\left(x_{i}\right)_{i \in \omega} \leq \pi\left(y_{i}\right)_{i \in \omega}$,

(I3) mixed associativity: for every infinite sequence $\left(x_{i}\right)_{i \in \omega} \in M^{\omega}$, for every $x \in M$, given $x_{i}^{\prime}=x$ when $i=0$ and $x_{i}^{\prime}=x_{i-1}$ when $i>0$, we have $\left(x \cdot\left(\pi\left(x_{i}\right)_{i \in \omega}\right)\right)^{R}=\pi\left(x_{i}^{\prime}\right)_{i \in \omega}$

(I4) infinite associativity: for every infinite sequence $\left(x_{i}\right)_{i \in \omega} \in M^{\omega}$, for every strictly increasing sequence $\left(k_{i}\right)_{i \in \omega}$ of positive integers with $k_{0}=0$, given $y_{i}=x_{k_{i}} \cdot x_{k_{i}+1} \cdots x_{k_{i+1}-1}$ defined for every $i \in \omega$, we have $\pi\left(y_{i}\right)_{i \in \omega}=$ $\pi\left(x_{i}\right)_{i \in \omega}$.

Definition 7 (Monoid completion). Let $S=\left(S_{f}, S_{\omega}\right)$ be an $\omega$-semigroup with finite product $\cdot: S_{f} \times S_{f} \rightarrow S_{f}$, mixed product $*: S_{f} \times S_{\omega} \rightarrow S_{\omega}$ and infinite product $\pi:\left(S_{f}\right)^{\omega} \rightarrow S_{\omega}$. By definition (see [30]) the finite, mixed and infinite product are related by mixed and infinite associativity laws. Let $S_{f}^{1}$ be the semigroup $S_{f}$ extended with a unit and let $\mathcal{P}^{*}\left(S_{\omega}\right)$ be the set of non-empty subsets of $S_{\omega}$.

The monoid completion $M(S)$ of the $\omega$-semigroup $S$ is defined to be the $M(S)=S_{f}^{1} \times \mathcal{P}^{*}\left(S_{\omega}\right)+0$ equipped with the product $\cdot$ defined, for every non zero element $(x, X)$ and $(y, Y) \in M(S)$ by $(x, X) \cdot(y, Y)=\left(x y, y^{-1}(X) \cap Y\right)$ when $y^{-1}(X) \cap Y \neq 0$ with $y^{-1}(X)=\left\{z \in S_{\omega}: y * z \in X\right\}$ and is defined to be 0 all other cases, with $y^{-1}(X)=\left\{z \in S_{\omega}: y * z \in X\right\}$. Element of $M(S)$ are also ordered by the relation $\leq$ defined over $M(S)$ by taking 0 to be the smallest element and by $(x, X) \leq(y, Y)$ when $x=y$ and $X \subseteq Y$ for every $(x, X)$ and $(y, Y) \in M(S)$.

Theorem 8. The set $M(S)$ with the above product - and the natural order $\leq$ is an $E$-ordered monoid with unit $1=\left(1, S_{\omega}\right)$.

Proof. The proof follows from the following Lemmas.

Lemma 9 (Soundness). The set $M(S)$ with the above product - and the natural order $\leq$ is a partially ordered monoid with unit $1=\left(1, S_{\omega}\right)$. 
Proof. Associativity and stability of the order relation are easily proved with essentially the same argument as in [14] though with subsets of $S_{\omega}$ instead of right ideals of $S_{f}$. The fact that $1=\left(1, S_{\omega}\right)$ makes no difficulty either.

Let $U(M(S))=\{x \in M(S): x \leq 1\}$ be the set of subunits of $M(S)$.

Lemma 10 (Adequacy). The set $M(S)$ is an adequately ordered monoid.

Proof. By definition, a subunit of $M(S)$ is either 0 or an element of the form $(1, X)$ for some $X \subseteq S_{\omega}$. It follows that (A1) holds: subunits are indeed idempotent elements.

Let us prove (A2). We easily check that $0^{L}=0^{R}=0$. Let $x \in M(S)$ be some non zero element of $M(S)$, that is an element of the form $x=(s, X)$. We prove that we have $x^{R}=(1, s * X)$ and its left projection $x^{L}=(1, X)$.

For the right projection, let $z=(1, s * X)$. We have $z \cdot x=\left(x, X \cap s^{-1}(s * X)\right)$ but $X \subseteq s^{-1}(s * X)$ hence $z \cdot x=x$. Now, if $z^{\prime} \cdot x=x$ for some $z^{\prime}=(1, Y)$ then we have $X=X \cap s^{-1}(Y)$ hence $X \subseteq s^{-1}(Y)$ hence $s * X \subseteq Y$ that is, following the order definition, $z \leq z^{\prime}$.

For the left projection, let $z=(1, X)$. We have $x \cdot z=\left(s, X \cap 1^{-1}(X)\right)=$ $(s, X)=z$. If $x \cdot z^{\prime}=x$ for some $z=(1, Y)$ we have $x \cdot z^{\prime}=\left(s, Y \cap 1^{-1}(X)\right)=$ $(s, X)$ hence $X \subseteq Y$ that is $z \leq z^{\prime}$.

Lemma 11 (Congruence property). The monoid $M(S)$ is even an E-ordered monoid.

Proof. We prove that $M(S)$ satisfies (A3). Let $x, y, z \in S$. If any of $x, y$ or $z$ is zero then the claim is satisfied. Assume $x=\left(x_{1}, X\right), y=\left(y_{1}, Y\right)$ and $z=\left(z_{1}, Z\right)$.

For the left projection, assume that $x^{L}=y^{L}$. This means that $X=Y$. We have $x z=\left(x_{1} z_{1}, z_{1}^{-1}(X) \cap Z\right)$ hence $(x z)^{L}=\left(1, z_{1}^{-1}(X) \cap Z\right)$. A similar computation shows that $(y z)^{L}=\left(1, z_{1}^{-1}(Y) \cap Z\right)$ hence the claim since $X=Y$.

For the right projection, assume that $x^{R}=y^{R}$. This means that $x_{1} * X=$ $y_{1} * Y$. We have $z x=\left(z_{1} x_{1}, x_{1}^{-1}(Z) \cap X\right)$ hence $(z x)^{R}=\left(1, z_{1} * x_{1} *\left(x_{1}^{-1}(Z) \cap X\right)\right)$. A similar computation shows that $(z y)^{R}=\left(1, z_{1} y_{1}\left(y_{1}^{-1}(Z) \cap Y\right)\right)$. Now let $z^{\prime} \in$ $z_{1} * x_{1} *\left(x_{1}^{-1}(Z) \cap X\right)$. By definition, and mixed associativity in $S$ hence we do not bother about parenthesis, this means there is $x^{\prime} \in X$ such that $z^{\prime}=z_{1} * x_{1} * x^{\prime}$ with the additional constraint that $x_{1} * x^{\prime} \in Z$ since $x^{\prime} \in x_{1}^{-1}(Z)$. But since $x_{1} * X=y_{1} * Y$, this means there exists $y^{\prime} \in Y$ such that $x_{1} * x^{\prime}=y_{1} * y^{\prime} \in Z$ hence $y^{\prime} \in y_{1}^{-1}(Z) \cap Y$ and thus $z^{\prime}=z_{1} * x_{1} * x^{\prime}=z_{1} * y_{1} * y^{\prime} \in z_{1} * y_{1} *\left(y_{1}^{-1}(Z) \cap Y\right)$. This proves that $(z y)^{R} \leq(z x)^{R}$. The reverse inequality is proved by applying a symmetrical argument.

This concludes the proof of Theorem 8 .

Remark. The notion of E-ordered monoid, extending Ehresmann semigroups [24], appears in [6]. It is examined quite in detail in [14]. There, a general construction, based on left and right ideals, is provided to embed every monoid $S$ into a non trivial adequately ordered monoid $\mathcal{Q}(S)$ : the quasi-inverse expansion of $S$ that 
turned out to be E-ordered as well. One can observe that the construction of $M(S)$ is quite similar with right ideals replaced by non empty subsets of $S_{\omega}$.

We define the infinite product $\pi$ almost by iteration although, as well known in $\omega$-language theory, the infinite product itself cannot be defined as a limit since many regula languages are not closed in prefix topology.

Among subunits of $M(S)$, the meet $\wedge$ in the order correspond to the product. Indeed, a subunit in $M(S)$ is either zero (with just behave like $(1, \emptyset)$ ) or of the form $(1, X)$ for some non empty $X \subseteq S_{\omega}$ with $(1, X) \cdot(1, Y)=(1, X \cap Y)$. The monoid of subunits $U(S)$ is thus isomorphic to the power set $\mathcal{P}\left(S_{\omega}\right)$ with intersection as product. In the next definition, when appropriate, we thus may use at will the meet operator $\wedge$ in place of the product.

Definition 12 (Infinite product). Let $\left(x_{i}\right)_{i \in \omega} \in(M(S))^{\omega}$. Let $\pi^{0}()=1$ and for every $n \in \omega$, let $\pi^{n+1}\left(x_{i}\right)_{i \leq n}=\left(x_{0} \cdot \pi^{n}\left(x_{i+1}\right)_{i<n}\right)^{R}$. Then, the infinite product $\pi\left(x_{i}\right)_{i \in \omega}$ is defined by

$$
\pi\left(x_{i}\right)_{i \in \omega}=\left(1, X_{\omega}\right) \wedge \bigwedge_{n \in \omega} \pi^{n}\left(x_{i}\right)_{i \leq n}
$$

with $X_{\omega}$ defined by $X_{\omega}=S_{\omega}$ when $J=\left\{i \in \omega: x_{i} \leq 1\right\}$ is finite and $X_{\omega}$ defined by $X_{\omega}=\left\{x_{\omega}\right\}$ when $J$ is infinite, with $x_{\omega}=\pi\left(s_{j_{i}}\right)_{i<\omega}$ where $\left(j_{i}\right)_{i \in \omega}$ is the increasing enumeration of the elements of $J$ and, for very $j \in J$, the (non zero) element $x_{j}$ is of the form $x_{j}=\left(s_{j}, X_{j}\right)$.

Theorem 13. The E-ordered monoid $M(S)$ equipped with the above infinite product is an adequately ordered $\omega$-monoid.

Proof. Subunit preservation (I1) immediately follows from the definition. Monotonicity (I2) is also easily check. Indeed, if $\left(x_{i}\right)_{i \leq \omega} \leq\left(y_{i}\right)_{i \in \omega}$ then, by definition of the order relation, this means that for every $i \in \omega$ if $x_{i}=\left(s_{i}, X_{i}\right)$ for some $s_{i} \in S_{f}$ and $X_{i} \subseteq S_{\omega}$ then we have $y_{i}=\left(s_{i}, Y_{i}\right)$ for some $Y_{i} \subseteq S_{\omega}$ with $X_{i} \subseteq Y_{i}$. It is then routine to check that we indeed have $\pi\left(x_{i}\right)_{i \leq \omega} \leq \pi\left(y_{i}\right)_{i \in \omega}$.

Mixed associativity (I3) quite immediately follows from Lemma 11 and the mixed associativity in $S$.

It remains to check that the product and the infinite product also satisfies the infinite associativity law (I4). Let $\left(x_{i}\right)_{i \in \omega} \in\left(S_{f} \times\left\{S_{\omega}\right\}\right)^{\omega}$, let $\left(j_{i}\right)_{i \in \omega}$ a strictly increasing sequence of integers with $j_{0}=0$, and, for every $i \in \omega$, let $y_{i}=x_{j_{i}} \cdot x_{j_{i}+1} \cdot \cdots \cdot x_{j_{i+1}-1}$. We have to prove that $\pi\left(x_{i}\right)_{i \in \omega}=\pi\left(y_{i}\right)_{i \in \omega}$. By definition, for every $i \in \omega$, we have

$$
\pi^{j_{i+1}}\left(x_{j}\right)_{j<j_{i+1}} \leq \pi^{i}\left(y_{j}\right)_{j<i} \leq \pi^{j_{i}}\left(x_{j}\right)_{j<j_{i}}
$$

hence $\bigwedge_{i \in \omega} \pi^{i}\left(x_{j}\right)_{j<i}=\bigwedge_{i \in \omega} \pi^{i}\left(y_{j}\right)_{j<i}$. In the case any of these meet is zero then we are done. Otherwise, assuming that, for every $i<\omega$ we have $x_{i}=\left(s_{i}, X_{i}\right)$ and $y_{i}=\left(t_{i}, Y_{i}\right)$ with $t_{i}=s_{j_{i}} \cdot s_{j_{i}+1} \cdots \cdots \cdot s_{j_{i+1}-1}$ we have to check that $X_{\omega}=Y_{\omega}$ as defined above. But this immediately follows from the definition and the fact $S$ is an $\omega$-semigroup. Indeed, either we have $X_{\omega}=Y_{\omega}=S_{\omega}$, or we have $X_{\omega}=\left\{x_{\omega}\right\}$ 
and $Y_{\omega}=\left\{y_{\omega}\right\}$ with $x_{\omega}=y_{\omega}$ that follows from the infinite associativity property in the $\omega$-semigroup $S$.

Last, we aim at showing that the $\omega$-monoid $S=\left(S_{f}, S_{\omega}\right)$ can be embedded as an $\omega$-monoid into $M(S)$. This means defining over $M(S)$ an $\omega$-monoid structure $\left(M_{f}(M), M_{\omega}(S)\right)$, which can be done by taking $M_{f}(S)=M(S)$ for the "finitary" part and $M_{\omega}(S)=U(M(S))$ for the "infinitary" part. The infinite product is the one already defined, and the mixed product of two elements $x \in M(S)$ and $y \in U(M(S))$ is defined to be $x * y=(x \cdot y)^{R}$.

Theorem 14. The mapping $\theta=\left(\theta_{f}, \theta_{\omega}\right):\left(S_{f}, S_{\omega}\right) \rightarrow(M(S), U(M(S)))$, defined by $\theta_{f}(x)=\left(x, S_{\omega}\right)$ for every $x \in S_{f}$ and by $\theta_{\omega}(y)=(1,\{y\})$ for every $y \in S_{\omega}$, is a one-to-one $\omega$-monoid morphism.

Proof. We first check that the pair of sets $(M(S), U(M(S)))$ equipped with the product $\cdot$, the mixed product $*$ and the infinite product $\pi$ is an $\omega$-semigroup.

Lemma 9 ensures that $M(S)$ equipped with the product · is a semigroup. The mixed associativity law follows from Lemma 11. The infinite associativity law follows from Theorem 13.

The fact $\theta_{f}$ is a one-to-one monoid morphism follows from the fact that for every $x \in S_{f}$ we have $x^{-1}\left(S_{\omega}\right)=\left\{y \in S_{\omega}: x * y \in S_{\omega}\right\}=S_{\omega}$ hence we indeed have $\theta_{f}(x \cdot y)=\theta_{f}(x) \cdot \theta_{f}(y)$ for every $x, y \in S_{f}$.

Let us show it preserves the mixed product. Let $x \in S_{f}$ and $y \in S_{\omega}$. We have $\theta(x * y)=(1,\{x * y\})$ and $\theta(x) * \theta(y)=\left(\left(x, S_{\omega}\right) \cdot(1,\{y\})\right)^{R}=((x,\{y\}))^{R}=$ $(1,\{x * y\})$ hence we indeed have equality. Observe that this property is also a particular case of the congruence property proved in Lemma 11.

It remains to prove that $\theta$ also preserves infinite products. Let $\left(x_{i}\right)_{i<\omega} \in$ $\left(S_{f}\right)^{\omega}$. By definition of $\theta_{\omega}$, we have $\theta_{\omega}\left(\pi\left(x_{i}\right)_{i \in \omega}\right)=\left(1,\left\{\pi\left(x_{i}\right)_{i \in \omega}\right\}\right)$. By definition of the infinite product, since $\theta_{f}\left(x_{i}\right) \not \leq 1$ for every $i \in \omega$, we have $\pi\left(\theta_{f}\left(x_{i}\right)\right)_{i \in \omega}=$ $\theta_{\omega}\left(\pi\left(x_{i}\right)_{i \in \omega}\right) \wedge \bigwedge_{i \in \omega} \pi^{i}\left(\theta\left(x_{j}\right)\right)_{j<i}$. Now, by definition of $\theta_{f}$, for every $i \in \omega$, we have $\theta_{f}\left(x_{i}\right)=\left(x_{i}, S_{\omega}\right)_{i \in \omega}$ hence, by definition of $\pi^{i}, \pi^{i+1} \theta\left(x_{j}\right)_{j \leq i}=\left(1, \bigcap_{j \leq i} x_{0} *\right.$ $\left.x_{1} * \cdots * x_{j} * S_{\omega}\right)$. Now, by mixed associativity in $S$, we have we have $\pi\left(x_{i}\right)_{i \in \omega} \in$ $x_{0} * x_{1} * \cdots * x_{i} * S_{\omega}$ for every $i \in \omega$, hence $\theta_{\omega}\left(\pi\left(x_{i}\right)_{i \in \omega}\right) \leq \pi^{i}\left(\theta\left(x_{j}\right)\right)_{j<i}$ henceforth $\pi\left(\theta_{f}\left(x_{i}\right)\right)_{i \in \omega}=\theta_{\omega}\left(\pi\left(x_{i}\right)_{i \in \omega}\right)$.

\section{Application to language theory}

We provide here a complete language theory for birooted words. Since every non-zero birooted word can just be seen as a pair composed of a finite and a finite or infinite word, the logical definability in Monadic Second-Order (MSO) logic of languages of birooted words boils down to the classical finite and infinite word language theory. More interestingly, we also provide a notion of finite-state birooted word automata and algebras that essentially captures definability in MSO logic.

A language of birooted words is a set $X \subseteq T^{\infty}(A)$ of non-zero birooted words. The class of such languages is equipped with the boolean operators union 
(also called sum), intersection and complement, plus operators derived from the structure of $T_{0}^{\infty}(A)$ : for all $X$ and $Y \subseteq T^{\infty}(A)$, the product $X \cdot X$, the star $X^{*}$, as well as $X^{+}$and the omega $X^{\omega}$, are defined by extending the corresponding operators on $T_{0}^{\infty}(A)$ in a point-wise manner, always omitting the birooted word zero possibly resulting from these products. Additionally, we define the left and right projections $X^{L}$ and $X^{R}$ of the language $X$ as the sets $X^{L}=\left\{x^{L} \in T^{\infty}(A)\right.$ : $x \in X\}$ and $X^{R}=\left\{x^{R} \in T^{\infty}(A): x \in X\right\}$.

Theorem 15. The class of languages of (positive) birooted words definable in $M S O$ is closed under all the operators defined above. Moreover, it is finitely generated from the finite languages of (positive) birooted words, sum, product, star, omega and left and right projections.

Proof. Easily follows from standard relativization techniques of mathematical logic.

Combining the notions of Muller $\omega$-word automata [28] (see also [30]) and of tile automata [18], we define birooted words $\omega$-automata as follows.

Definition 16. A (finite) birooted word $\omega$-automaton is a tuple $\mathcal{A}=\langle Q, \delta, K, W\rangle$ with a (finite) set of states $Q$, a transition function $\delta: A \rightarrow \mathcal{P}(Q \times Q)$, a finitary acceptance condition $K \subseteq Q \times Q$ and an infinitary acceptance condition $W \subseteq \mathcal{P}(Q)$. For technical reasons, we always assume that $\emptyset \in W$.

A run of the automaton $\mathcal{A}$ on a birooted word $u=\left(u_{1}, u_{2}\right)$ is a labeling mapping $\rho:\left[0,\left|u_{1} * u_{2}\right|+1[\rightarrow Q\right.$ that satisfies the local consistency property : $(\rho(k), \rho(k+1)) \in \delta\left(\left(u_{1} * u_{2}\right)[k]\right)$ for every $0 \leq k \leq\left|u_{1}\right|+\left|u_{2}\right|$.

The run $\rho$ of the automaton $\mathcal{A}$ on the birooted word $u=\left(u_{1}, u_{2}\right)$ is locally accepting when the pair of states $\left(\rho(0), \rho\left(\left|u_{1}\right|\right)\right)$ that marks the input and output roots belongs to $K$.

The run $\rho$ is globally accepting when the set of states that occur infinitely often belongs to $W$, i.e. $\left.\left\{q \in Q:\left|\varphi^{-1}(q)\right|=\infty\right\} \in W\right\}$. Observe that when $\left(u_{1}, u_{2}\right)$ is finite then the global acceptance constraint is always satisfied since we assume that $\emptyset \in W$.

The language $L(\mathcal{A})$ is then defined as the set of birooted words $\left(u_{1}, u_{2}\right) \in$ $T^{\infty}(A)$ for which there exists a locally and globally accepting run of $\mathcal{A}$ on $\left(u_{1}, u_{2}\right)$.

Theorem 17. A language $L \subseteq T^{\infty}(A)$ of non nul birooted words is recognized by a finite-state birooted word $\omega$-automaton if and only if $L$ is definable in MSO and upward closed.

Proof. Assume that $L$ is recognizable by a finite-state automaton. Since the existence of an accepting run on a birooted word amounts to checking the existence of some labeling of the vertices of (the graph representation of) the birooted word, this property is classically definable in MSO. Then, by definition of birooted word automata, the language $L$ is upward closed w.r.t. in the birooted word order. 
Conversely, assume that $L$ is definable in MSO. By applying classical automata theoretic techniques, there exists an finite-state word automaton $\mathcal{A}^{\prime}$ recognizing the language of finite and infinite words $L^{\prime}=\left\{u_{1} \# u_{2} \in(A+\#)^{\infty}\right.$ : $\left.\left(u_{1}, u_{2}\right) \in L\right\}$. It is not difficult to derive from the automaton $\mathcal{A}^{\prime}$ a birooted word automaton $\mathcal{A}$ recognizing, under upward closure hypothesis, the language $L$.

Corollary 18. The class of languages recognized by a finite-state birooted word $\omega$-automaton is closed under the sum, intersection, product, star and omega operations. Moreover, it is finitely generated from finite languages of (positive) birooted words, sum, product, star and omega, and the upward closure of left and right projections.

The next definitions and theorem extend a similar result proved in [18] for languages of finite birooted words.

A mapping $\varphi: T_{0}^{\infty}(A) \rightarrow T$ from birooted words to an adequately ordered monoid $T$ is a premorphism when $\varphi(1)=1$ and $\varphi(x y) \leq \varphi(x) \varphi(y)$ and if $x \leq y$ then $\varphi(x) \leq \varphi(y)$ for every $x$ and $y \in T_{0}^{\infty}(A)$.

The premorphism $\varphi$ is adequate when, moreover, $\varphi\left(x^{L}\right)=(\varphi(x))^{L}$ and $\varphi\left(x^{R}\right)=(\varphi(x))^{R}$ for every $x \in S$, and if $x^{L} y^{R} \neq 0$ and $x^{L} \vee y^{R}=1$ then $\varphi(x y)=\varphi(x) \varphi(y)$ for every $x$ and $y \in S$. In the latter case we say that the product $x y$ is disjoint.

The adequate premorphism $\varphi$ is $\omega$-adequate when $T$ is an adequately ordered $\omega$-monoid and, for every infinite sequence $\left(u_{i}\right)_{0 \leq i} \in\left(T_{\infty}^{0}(A)\right)^{\omega}$, we have $\varphi\left(\pi\left(u_{i}\right)_{0 \leq i}\right) \leq \pi\left(\varphi\left(u_{i}\right)\right)_{0 \leq i}$ and, moreover, if the product $\pi\left(u_{i}\right)_{0 \leq i}$ is disjoint (i.e. for every $0<k$, given $x=u_{0} \cdot u_{1} \cdots u_{k-1}$ and $y=\pi\left(u_{i+k}\right)_{0 \leq i}$ we have $x^{L} y^{R} \neq 0$ and $\left.x^{L} \vee y^{R}=1\right)$, then we also have $\varphi\left(\pi\left(u_{i}\right)_{0 \leq i}\right)=\pi\left(\varphi\left(u_{i}\right)\right)_{0 \leq i}$.

A language $L \subseteq T^{\infty}(A)$ is quasi-recognizable when there exists a finite adequately ordered $\omega$-monoid $S$ and an $\omega$-adequate premorphism $\varphi: T_{0}^{\infty}(A) \rightarrow S$ such that $L=\varphi^{-1}(\varphi(T))$.

Theorem 19. Let $L \subseteq T_{0}^{\infty}(A)$ be a language of non-zero finite or infinite birooted words. The language $L$ is quasi-recognizable if and only if it is a finite boolean combination of upward-closed (for the natural order) languages definable in monadic second order logic (MSO).

Proof. Assume that there is an $\omega$-adequate premorphism $\varphi: T_{0}^{\infty}(A) \rightarrow S$ that recognizes $L$. Without loss of generality, possibly by adding a new zero to $S$, we may assume that $\varphi^{-1}(0)=0$. Then, for every non-zero $s \in S, \varphi^{-1}(s)$ is definable in MSO.

Indeed, the mapping that maps every finite word $u \in A^{*}$ to $\varphi((u, 1))$ is a monoid morphism since, for every $a \in A$, every $u \in A^{*}$, the product $(a, 1) \cdot(u, 1)$ is disjoint. Then, given $\left(u_{1}, u_{2}\right) \in T^{\infty}(A)$, since $\left(u_{1}, u_{2}\right)=\left(u_{1}, 1\right) \cdot\left(1, u_{2}\right)$ with a disjoint product, we have $\varphi\left(\left(u_{1}, u_{2}\right)\right)=\varphi\left(\left(u_{1}, 1\right)\right) \cdot\left(\varphi\left(1, u_{2}\right)\right)$. In the case $u_{2}$ is also finite, we are done since $\varphi\left(1, u_{2}\right)=\varphi\left(\left(u_{2}, 1\right)^{R}\right)=\varphi\left(\left(u_{2}, 1\right)\right)^{R}$. In the case when $u_{2}$ is infinite, we can apply Ramsey's theorem and infinite associativity, following the technique used for infinite words [30], to show that $\left(1, u_{2}\right)=\pi\left(\left(v_{i}, 1\right)\right)_{0 \leq i}$ with $\varphi\left(\left(v_{i}, 1\right)\right)=\varphi\left(\left(v_{1}, 1\right)\right)$ henceforth $\varphi\left(\left(1, u_{2}\right)\right)=\left(\varphi\left(\left(v_{0}, 1\right)\right) \cdot\left(\varphi\left(\left(v_{1}, 1\right)\right)\right)^{\omega}\right)^{R}$. 
Then, by applying classical arguments of algebraic language theory, the fact that $L$ is a finite boolean combination of upward closed languages is a consequence of the monotonicity of $\varphi$ and the finiteness of $S$ since, for every $s \in \varphi(L)$, we have $\varphi^{-1}(s)=\varphi^{-1}\left(s^{\uparrow}\right) \cap \varphi^{-1}\left(s^{\downarrow}\right)$ with $s^{\uparrow}$ (resp. $s^{\downarrow}$ ) is the upward closure (downward closure) of the singleton $\{s\}$.

Conversely, assume that $L$ is a finite boolean combination of upward closed languages $\left\{L_{i}\right\}_{0 \leq i \leq n}$ definable in MSO. For every $0 \leq i \leq n$, by applying Theorem 17 , the language $L_{i}$ is recognized by a finite-state automaton $\mathcal{A}_{i}$. Now, by applying Lemma 20 below, for every $i \in I$, there exists an $\omega$-adequate premorphisms $\varphi_{i}: T_{0}^{\infty}(A) \rightarrow S_{i}$ with finite $S_{i}$ such that $L_{i}=\varphi_{i}^{-1}\left(\varphi_{i}\left(L_{i}\right)\right)$ hence we conclude by applying classical algebraic techniques that the product premorphism

$$
\varphi: T_{0}^{\infty}(A) \rightarrow S=S_{0} \times S_{1} \times \cdots \times S_{n}
$$

defined for every $x \in T_{0}^{\infty}(A)$ by

$$
\varphi(x)=\left(\varphi_{0}(x), \varphi_{1}(x), \cdots, \varphi_{n}(x)\right)
$$

quasi-recognized $L$. Proving that the product monoid $S$ is a (finite) adequately ordered $\omega$-monoid raises no particular difficulties.

Lemma 20. Let $\mathcal{A}$ be a finite-state birooted word $\omega$-automaton. There exists a (finite) adequately ordered $\omega$-monoid $S_{\mathcal{A}}$ and an $\omega$-adequate premorphism $\varphi_{\mathcal{A}}$ : $T_{0}^{\infty}(A) \rightarrow S_{\mathcal{A}}$ such that $L(\mathcal{A})=\varphi_{\mathcal{A}}^{-1}\left(\varphi_{\mathcal{A}}(L(\mathcal{A}))\right.$.

Proof. Let $\mathcal{A}=\langle Q, \delta, K, W\rangle$ be the automaton. Let $T_{Q} \subseteq Q \times \mathcal{P}(Q) \times Q \times \mathcal{P}(Q)$ be the set of automaton traces, that is, quadruples of the from $(p, P, q, R) \in$ $Q \times \mathcal{P}(Q) \times Q \times \mathcal{P}(Q)$ with $p \in P$ when $p \neq q$, with, intendedly, a input root state $p$, a set of states $P$ occurring between the input and the output root, an output root state $q$ and a set of states $R$ occurring infinitely often.

The set $T_{Q}$ is equipped with the partial product - defined, for every $(p, P, q, R)$ and $\left(p^{\prime}, P^{\prime}, q^{\prime}, R^{\prime}\right) \in T_{Q}$, when $q=p^{\prime}$ and either $R=\emptyset$ or $R^{\prime}=\emptyset$ or $R=R^{\prime}$, by

$$
(p, P, q, R) \cdot\left(p^{\prime}, P^{\prime}, q^{\prime}, R^{\prime}\right)=\left(p, P \cup P^{\prime}, q^{\prime}, R \cup R^{\prime}\right)
$$

One can check that, although partial, it is nevertheless associative in the sense that for every traces $t, t^{\prime}, t^{\prime \prime}$, we have the product $\left(t \cdot t^{\prime}\right) \cdot t^{\prime \prime}$ is defined if and only if the product $t \cdot\left(t^{\prime} \cdot t^{\prime \prime}\right)$ is defined and, in that case, they are equal.

The set $T_{Q}$ is also equipped with an infinite product $\pi$ defined, for every sequence $\left(p_{i}, P_{i}, q_{i}, R_{i}\right)_{0 \leq i}$ of traces of $T_{Q}$, when $q_{i}=p_{i+1}$ and $R_{i}=\emptyset$ for every $0 \leq i$, by

$$
\pi\left(p_{i}, P_{i}, q_{i}, \emptyset\right)_{0 \leq i}=\left(p_{0},\left\{p_{0}\right\}, p_{0}, R\right)
$$

with $R=\left\{q \in Q:\left|\left\{i \in \omega: q \in P_{i}\right\}\right|=\infty\right\}$.

Then we define $S_{\mathcal{A}}$ as the power set of $T_{Q}$, with product and infinite product defined as the point-wise extensions of the product and infinite product of $T_{Q}$. That is, for every $X$ and $Y \subseteq T_{Q}$, we define $X \cdot Y$ as the set of all defined products $x \cdot y$ in $T_{Q}$ with $x \in X$ and $y \in Y$, and, for every sequence $\left(X_{i}\right)_{0 \leq i}$ 
of subsets of traces, we define $\pi\left(X_{i}\right)_{0 \leq i}$ as the set of all defined products of the form $\pi\left(x_{i}\right)_{0 \leq i}$ with $x_{i} \in X_{i}$ for every $0 \leq i$.

It is routine to check that the monoid $S_{\mathcal{A}}$ obtained, ordered by inclusion, is an adequately ordered $\omega$-monoid (following the technicalities used in $[18,16]$ for finite birooted words or trees).

We define $\varphi_{\mathcal{A}}: T_{0}^{\infty}(A) \rightarrow S_{\mathcal{A}}$ by $\varphi(0)=\emptyset$ and, for all birooted words $\left(u_{1}, u_{2}\right)$, $\varphi\left(\left(u_{1}, u_{2}\right)\right)$ is the set of traces of the form $(p, P, q, R)$ such that there exists a run $\rho$ of the automaton $\mathcal{A}$ on $\left(u_{1}, u_{2}\right)$ with $\rho(0)=p, P=\rho\left(\left[0,\left|u_{1}\right|\right]\right), q=\rho\left(\left|u_{1}\right|\right)$ and $R=\left\{r \in|Q|:\left|\rho^{-1}(q)\right|=\infty\right\}$. Again, it is routine to check that $\varphi_{\mathcal{A}}$ is an $\omega$-adequate premorphism.

We finally check that, by construction, a birooted word $x \in T_{0}^{\infty}(A)$ belongs to $L$ if and only if there exist $(p, P, q, R) \in \varphi_{\mathcal{A}}(x)$ such that $(p, q) \in K$ and $R \in W$.

\section{Conclusion}

We have shown that the embedding of strings and streams into tiled streams, as done for programming purposes [21], can be lifted to arbitrary $\omega$-semigroups, thus unifying the finite and infinite word language theory into the emerging language theory of birooted words.

Since regular languages of finite and infinite tiled streams can be used as programming language subtypes, this result is also a new step in developing the idea that, in the long term, inverse semigroups [25] may be tuned towards robust engineering practice.

Following $[16,19]$, we could also aim at generalizing the present approach to trees, possibly leading to further developments in the very subtle and difficult emerging algebraic theory of languages of infinite trees [2]. However, there is no evidence yet that such a generalization could lead to a successful algebraic characterization of infinite tree languages.

\section{References}

1. F. Berthaut, D. Janin, and B. Martin. Advanced synchronization of audio or symbolic musical patterns: an algebraic approach. International Journal of Semantic Computing, 6(4):409-427, 2012.

2. A. Blumensath. Recognisability for algebras of infinite trees. Theoretical Comp. Science, 412(29):3463-3486, 2011.

3. J. R. Büchi. On a decision method in restricted second order arithmetic. In International Congress on Logic, Methodology and Philosophy of Science, pages 1-11. Standford University Press, 1962.

4. A. Dicky and D. Janin. Two-way automata and regular languages of overlapping tiles. Research report RR-1463-12, LaBRI, Université de Bordeaux, 2013.

5. A. Dicky and D. Janin. Embedding finite and infinite words into overlapping tiles. In Developments in Language Theory (DLT), LNCS, Ekaterinburg, Russia, 2014 (in print). Springer. 
6. E. Dubourg and D. Janin. Algebraic tools for the overlapping tile product. In Language and Automata Theory and Applications (LATA), volume 8370 of LNCS, pages 335 - 346. Springer, 2014.

7. J. Fountain, G. Gomes, and V. Gould. A Munn type representation for a class of E-semiadequate semigroups. Journal of Algebra, 218:693-714, 1999.

8. J. Fountain, G. Gomes, and V. Gould. The free ample monoid. Int. Jour. of Algebra and Computation, 19:527-554, 2009.

9. E. Grädel, W. Thomas, and T. Wilke, editors. Automata, Logics and Infinite Games, volume 2500 of LNCS Tutorial. Springer, 2002.

10. P. Hudak. A sound and complete axiomatization of polymorphic temporal media. Technical Report RR-1259, Department of Computer Science, Yale University, 2008.

11. P. Hudak. The Haskell School of Music : From signals to Symphonies. Yale University, Department of Computer Science, 2013.

12. P. Hudak, J. Hugues, S. Peyton Jones, and P. Wadler. A history of Haskell: Being lazy with class. In Third ACM SIGPLAN History of Programming Languages (HOPL). ACM Press, 2007.

13. P. Hudak and D. Janin. Tiled polymorphic temporal media. Research report RR-1478-14, LaBRI, Université de Bordeaux, 2014.

14. D. Janin. Quasi-inverse monoids (and premorphisms). Research report RR-145912, LaBRI, Université de Bordeaux, 2012.

15. D. Janin. Quasi-recognizable vs MSO definable languages of one-dimensional overlapping tiles. In Mathematical Found. of Comp. Science (MFCS), volume 7464 of LNCS, pages 516-528, 2012.

16. D. Janin. Algebras, automata and logic for languages of labeled birooted trees. In Int. Col. on Aut., Lang. and Programming (ICALP), volume 7966 of LNCS, pages 318-329. Springer, 2013.

17. D. Janin. On languages of one-dimensional overlapping tiles. In Int. Conf. on Current Trends in Theo. and Prac. of Comp. Science (SOFSEM), volume 7741 of $L N C S$, pages 244-256. Springer, 2013.

18. D. Janin. Overlaping tile automata. In 8th International Computer Science Symposium in Russia (CSR), volume 7913 of LNCS, pages 431-443. Springer, 2013.

19. D. Janin. On languages of labeled birooted trees: Algebras, automata and logic. Information and Computation, (in print), 2014.

20. D. Janin. Towards a higher dimensional string theory for the modeling of computerized systems. In Int. Conf. on Current Trends in Theo. and Prac. of Comp. Science (SOFSEM), volume 8327 of LNCS, pages 7-20. Springer, 2014.

21. D. Janin, F. Berthaut, M. DeSainte-Catherine, Y. Orlarey, and S. Salvati. The T-calculus : towards a structured programming of (musical) time and space. In ACM Workshop on Functional Art, Music, Modeling and Design (FARM), pages 23-34. ACM Press, 2013.

22. J. Kellendonk. The local structure of tilings and their integer group of coinvariants. Comm. Math. Phys., 187:115-157, 1997.

23. J. Kellendonk and M. V. Lawson. Tiling semigroups. Journal of Algebra, 224(1):140 $-150,2000$.

24. M. V. Lawson. Semigroups and ordered categories. I. the reduced case. Journal of Algebra, 141(2):422 - 462, 1991.

25. M. V. Lawson. Inverse Semigroups : The theory of partial symmetries. World Scientific, 1998.

26. M. V. Lawson. McAlister semigroups. Journal of Algebra, 202(1):276 - 294, 1998. 
27. D.B. McAlister. Inverse semigroups which are separated over a subsemigroups. Trans. Amer. Math. Soc., 182:85-117, 1973.

28. D. Muller. Infinite sequences and finite machines. In Fourth Annual Symp. IEEE, Switching Theory and Logical Design, pages 3-16, 1963.

29. M. Nivat and J.-F. Perrot. Une généralisation du monoïde bicyclique. Comptes Rendus de l'Académie des Sciences de Paris, 271:824-827, 1970.

30. D. Perrin and J.-E. Pin. Infinite Words: Automata, Semigroups, Logic and Games, volume 141 of Pure and Applied Mathematics. Elsevier, 2004. 